Supporting information

\title{
Bio-inspired ice growth inhibitors based on self-assembling peptides
}

\author{
Bin Xue ${ }^{\dagger}$, Lishan Zhao ${ }^{\ddagger}$, Xuehua Qin§, Meng Qin ${ }^{\dagger}$, Jiancheng Lai', Wenmao \\ Huang $^{\dagger}$, Hai Lei ${ }^{\dagger}$, Jianjun Wang ${ }^{*}$, Wei Wang ${ }^{\star \dagger}$, Ying Li $\left.{ }^{\star}\right\lrcorner$ and Yi Cao ${ }^{\dagger}$
}

+ Collaborative Innovation Center of Advanced Microstructures, National Laboratory of Solid State Microstructure, Key Laboratory of Intelligent Optical Sensing and Manipulation, Ministry of Education, Department of Physics, Nanjing University, Nanjing 210093, P.R. China

₹ Key Laboratory of Green Printing, Institute of Chemistry, Chinese Academy of Sciences, Beijing 10019o, P.R. China

$\$^{\S}$ College of Life Sciences and Health, Northeastern University, Shenyang 110169, P. R. China

" State Key Laboratory of Coordination Chemistry, Nanjing National Laboratory of Microstructures, Collaborative Innovation Center of Advanced Microstructures, School of Chemistry and Chemical Engineering, Nanjing University, Nanjing 210093, P. R. China.

${ }^{\perp}$ Collaborative Innovation Center of Atmospheric Environment and Equipment Technology, Jiangsu School of Environmental Science and Engineering, Nanjing University of Information Science \& Technology, Nanjing 210044, P.R. China

\section{Experimental Procedures}

Materials: All the peptides were purchased from GL Biochem (Shanghai) Ltd. The catalase assay kit was purchased from Beyotime Institute of Biotechnology, China. The lysozyme assay kit (HY-6oo61) was purchased from Shanghai Yubo Biological Technology Co., Ltd. Cell Counting Kit-8 (CCK-8) was purchased from KeyGEN BioTECH, China. Lysozyme powder from Hen Egg White ( 10oooo U mg $\left.{ }^{-1}\right)$, catalase solutions ( $\left.\geq 30,000 \mathrm{U} \mathrm{mg}^{-1}\right)$ from bovine liver, Poly(ethylene glycol) (PEG) $(7.5 \mathrm{kDa})$ and deuteroxide $\left(\mathrm{D}_{2} \mathrm{O}\right)$ were purchased form Sigma-Aldrich. Glycerol and dimethyl sulfoxide (DMSO) were purchased from J\&K. DMEM medium, Hyclone (FBS), penicillin streptomycin, trypsin and EDTA were purchased from Gibco, USA. Phosphate-buffered saline (PBS) solution was prepared using preformulated tablets (Sigma-Aldrich) in 200 $\mathrm{mL}$ of Milli-Q water $(>18.2 \Omega$ mean resistivity) to give $[\mathrm{NaCl}]=0.138 \mathrm{M},[\mathrm{KCl}]=0.0027 \mathrm{M}$, and $\mathrm{pH}$ 7.4. All reagents were used as received unless otherwise stated. 
Atomic Force Microscopy: The microscopic morphologies of the peptides were imaged at room temperature $\left(25^{\circ} \mathrm{C}\right)$ by a NanoWizard II AFM (JPK, Germany) operating in the intermittent contact (Air) mode (conditions: scan rate, $1 \mathrm{~Hz}$; pixel number, $512 \times 512$ ). Silica cantilevers (SSS-SeIHR-50, Nanosensors, Switzerland) with typical tip radii of $\sim 2-10 \mathrm{~nm}$ and resonance frequencies of $\sim 96$ to $175 \mathrm{kHz}$ were used for imaging. The AFM samples were prepared as described below: $50 \mu \mathrm{L}$ of peptide solutions (100 $\mu \mathrm{M})$ was loaded to a freshly peeled mica surface and allowed to adsorb for $10 \mathrm{~min}$. Then, the majority of solution was carefully removed and the mica surface was allowed to dry at room temperature $\left(25^{\circ} \mathrm{C}\right)$. The surface was further blown with compressed air to remove any loosely attached stuff prior to the AFM measurement.

The DSC measurement: The supercooling points and melting points of all the samples were measured using a differential scanning calorimeter (DSC Q10o; TA instruments, U.S.A.). For the supercooling point measurements, the temperature of the sample stage was lowered by $0.1{ }^{\circ} \mathrm{C} \min ^{-1}$ from o ${ }^{\circ} \mathrm{C}$ to $-35^{\circ} \mathrm{C}$. For the melting points measurements, the temperature was lowered by $0.1{ }^{\circ} \mathrm{C} \min ^{-1}$ from o ${ }^{\circ} \mathrm{C}$ to $-35{ }^{\circ} \mathrm{C}$ and then increased to $5{ }^{\circ} \mathrm{C}$. The concentration of the samples were all $1 \mathrm{mM}$. The supercooling point was defined as the intersection of the tangent line drawn at the increase of the exothermic signal with an extrapolated baseline. The melting point was defined by the intersection of the tangent line drawn at the inflection of the endothermic signal during the phase change with an extrapolated baseline. It is worth to mentioning that the DSC measurement cannot fully support that the peptide has ice-inhibition activity as many additives, such as PEG and DMSO, were demonstrated to show similar effects but had no ice binding activity.

Preparation of the self-assembly of KLVFGAK and KLVFFGAT peptides: KLVFGAK and KLVFFGAT peptides were dissolved in acidic PBS buffer $(50 \mathrm{mM}, \mathrm{pH}=\mathbf{2 . 0})$ to the concentration of $1 \mathrm{mM}$. Then the peptide solutions were incubated at $37{ }^{\circ} \mathrm{C}$ with constant agitation for $3 \mathrm{~d}$ and the nanostructures were formed. The DSC of KLVFGAK and KLVFFGAT peptides was performed at pH 2.o.

The cytotoxicity of peptides in SMMC-7721 and L-O2 cells: SMMC-7721 and L-O2 cells were seeded in a 24-well plate at a density of $1 \times 10^{4}$ cells per well (with o.8 mL DMEM medium). After 24 h incubation, 200 $\mu \mathrm{L}$ of medium containing different concentration of peptides were added to the wells with cells. Then all 
the cells were cultured for additional $48 \mathrm{~h}$. The cell viability was assessed by the MTT method. Firstly, cells were incubated with MTT (o.5 $\left.\mathrm{mg} \mathrm{mL}^{-1}\right)$ for $4 \mathrm{~h}$ at $37^{\circ} \mathrm{C}$. During this incubation period, water-insoluble crystals were formed and were then dissolved by the addition of $100 \mu \mathrm{L}$ DMSO per well. The optical densities at $570 \mathrm{~nm}$ were measured using an enzyme-linked immunosorbent assay plate reader. Wells containing culture medium and MTT but no cells acted as blanks. The percentage of cell viability was calculated as follows: $\frac{A_{\text {peptide }}-A_{\text {blank }}}{A_{\text {control }}-A_{\text {blank }}} \times 100 \%$.

The protection of bacteria with ice growth inhibiting peptides: Freshly prepared E.coli (BL21) cultures was diluted into PBS (10 mM, pH = 7.4) containing different concentrations of designed peptides or glycerol to a final $\mathrm{OD}_{6 \circ \mathrm{nm}}$ of o.1. The samples were snap frozen in $-80{ }^{\circ} \mathrm{C}$ deep freezer for $12 \mathrm{~h}$ before thawing at $25^{\circ} \mathrm{C}$ in a water bath for $5 \mathrm{~min}$. The freeze-thaw cycle was repeated for three times. For the growth curve of the freeze-thaw treated bacteria, $10 \mu \mathrm{L}$ of bacteria solutions after three freeze-thaw cycles were added into $5 \mathrm{~mL}$ of LB media and then the solutions were incubated at $37^{\circ} \mathrm{C}$ on an orbital shaker at a vibrating rate of $225 \mathrm{rpm}$. The $\mathrm{OD}$ value at $600 \mathrm{~nm}\left(\mathrm{OD}_{600} \mathrm{~nm}\right)$ was measured using a V-550 (JASCO Inc., Japan) UV-vis spectrophotometer every hour to monitor the growth of bacteria. The cuvette width was $1 \mathrm{~mm}$ and the bandwidth was $0.2 \mathrm{~nm}$.

For the growth of bacteria on agar plates ( $\mathrm{LB}$ media $+2 \%(\mathrm{w} / \mathrm{w})$ agar), $\sim 150 \mu \mathrm{L}$ of bacteria suspensions after three freeze-thaw cycles was plated on the agar plates evenly. Then the agar plates were grown for $15 \mathrm{~h}$ at $37^{\circ} \mathrm{C}$ in an incubator before being imaged. The number of colonies were counted by ImageJ. 


\section{Results and Discussion}
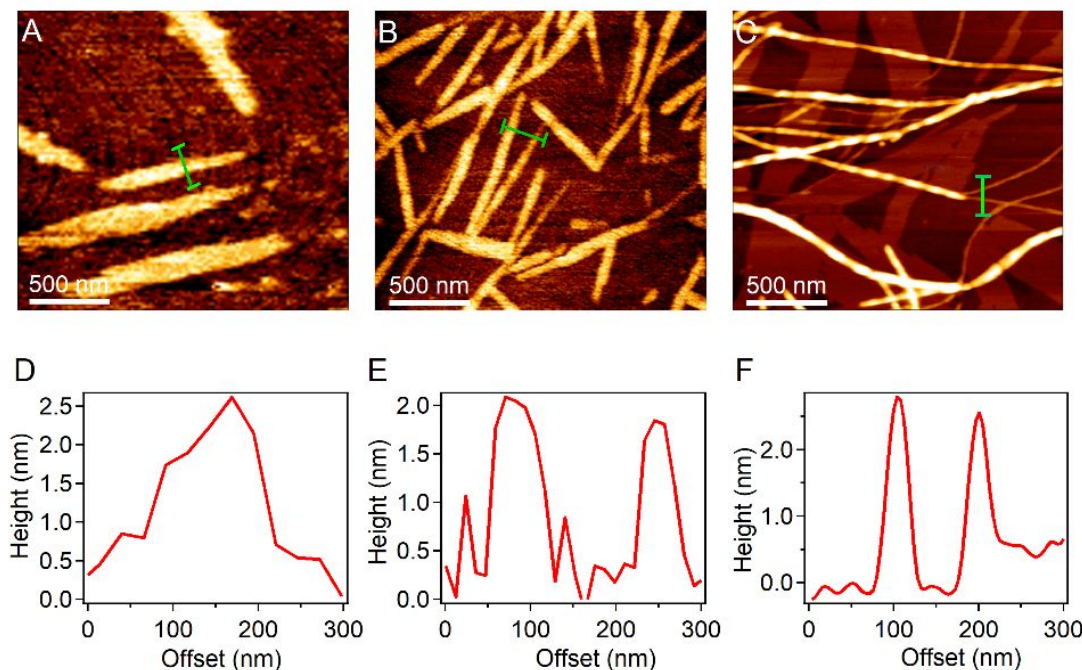

Figure S1 (A) AFM images of microstructures based on the self-assembly of 2-NapGFFT peptide. (B) AFM images of microstructures based on the self-assembly of 2-NapGFFTT peptide. (C) AFM images of microstructures based on the self-assembly of 2-NapGFFD peptide. (D) The heights of the cross sections marked in green in (A). (E) The heights of the cross sections marked in green in (B). (F) The heights of the cross sections marked in green in $(C)$.
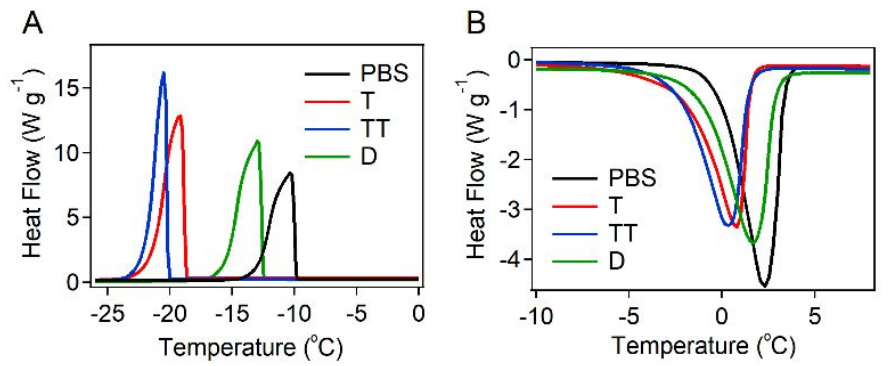

Figure S2 (A-B) DSC traces for 2-NapGFFT, 2-NapGFFTT, and 2-NapGFFD peptide solutions (1 mM) with PBS (10 mM, pH = 7.4) as the negative control. (A) Cooling; (B) Heating. PBS, T, TT and D correspond to PBS buffer (1o mM, PH=7.4), 2-NapGFFT, 2-NapGFFTT and 2-NapGFFD peptide solutions, respectively. 
A

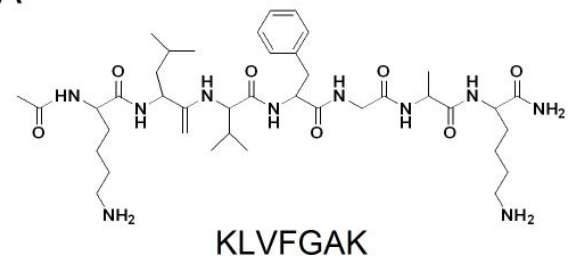

C

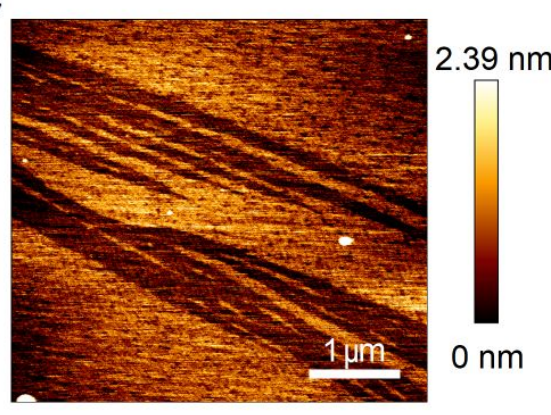

B

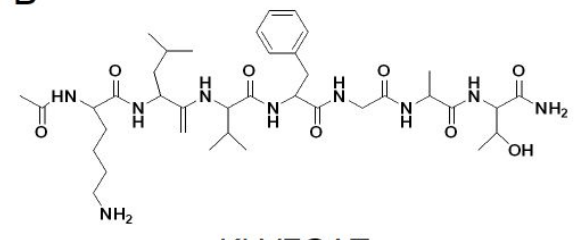

KLVFGAT

D

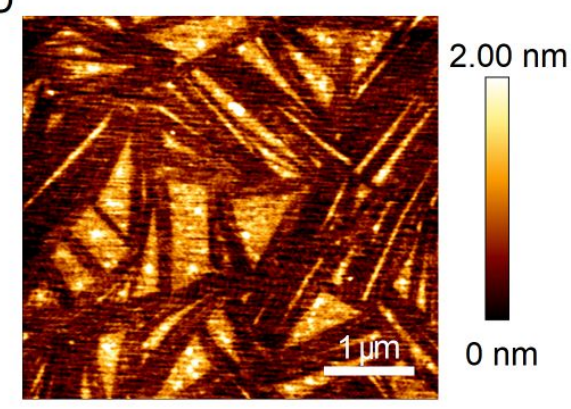

Figure $S_{3}$ (A) The chemical structure of KLVFGAK peptides. (B) The chemical structure of KLVFGAT peptides. (C) The AFM image of the self-assembled KLVFGAK peptide. (D) The AFM image of the selfassembled KLVFGAT peptide.
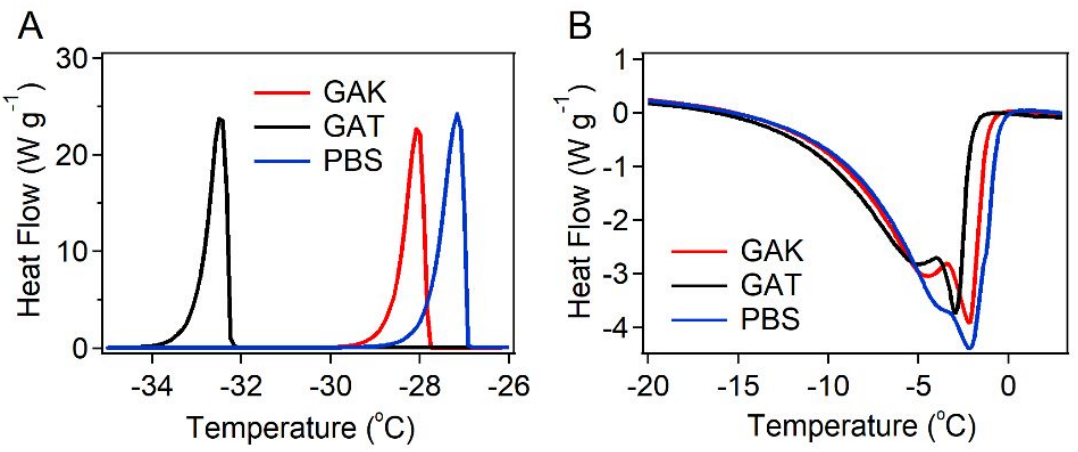

Figure $\mathrm{S}_{4}$ DSC traces for KLVFGAK and KLVFGAT peptide solutions ( $1 \mathrm{mM}$ ) with PBS (5o M, $\mathrm{pH}=2.0$ ) as the negative control. (A) Cooling; (B) Heating. PBS, GAK and GAT correspond to PBS buffer (50 mM, pH = 2.o), KLVFGAK and KLVFGAT peptide solutions, respectively.

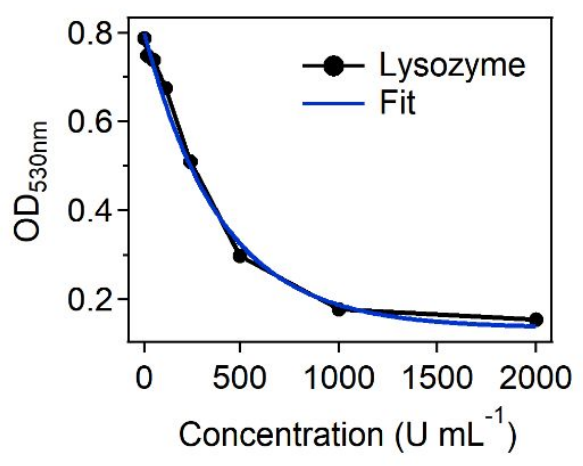

Figure $\mathrm{S}_{5}$ Calibration curve of lysozyme activities and $\mathrm{OD}_{53}$ onm in lysozyme solutions. 

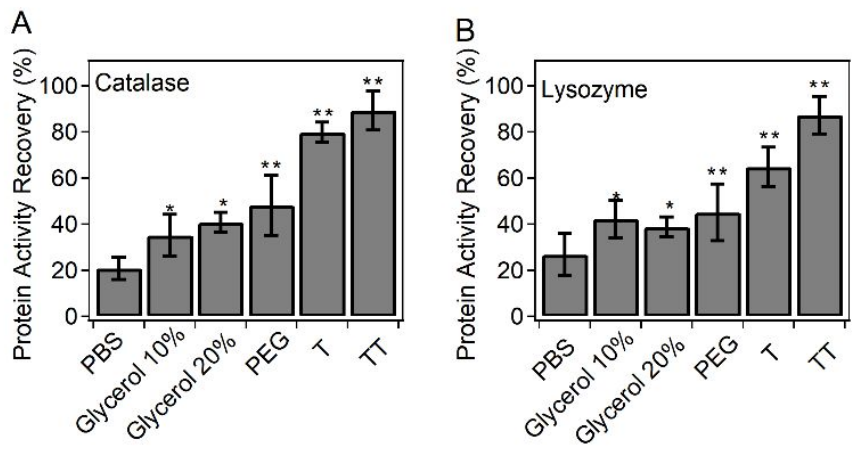

Figure S6 (A) Recovery of catalase activity after three freeze-thaw cycles as \% of fresh, unfrozen protein. Error bars are S. D. from 3 repeats. * represents $\mathrm{p}<0.05$ relative to PBS (10 mM, pH = 7.4) buffer control. ${ }^{* *}$ represents $\mathrm{p}<0.01$ relative to PBS (1o $\mathrm{mM}, \mathrm{pH}=7.4$ ) buffer control. The concentration of PEG (MW: 7.5 $\mathrm{kDa}$ ) was $100 \mathrm{mg} \mathrm{mL}^{-1}$ while that of glycerol was $10 \% \mathrm{w} / \mathrm{v}$ and $20 \% \mathrm{w} / \mathrm{v}$. The concetrations of NapGFFT and NapGFFTT were both $0.25 \mathrm{mg} \mathrm{mL}^{-1}$.
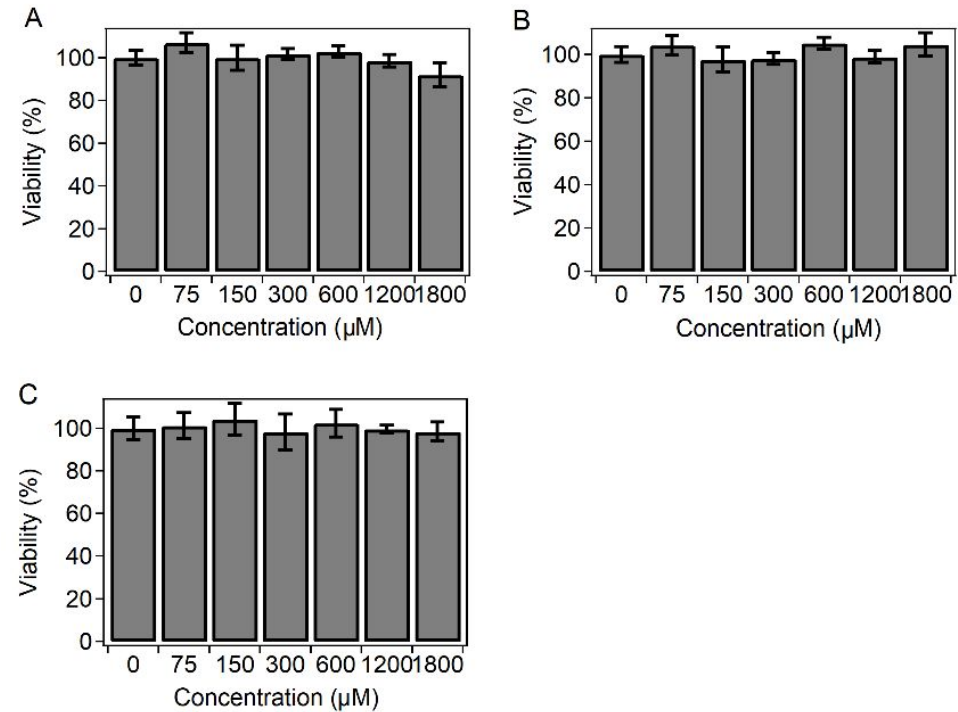

Figure $\mathrm{S}_{7}$ (A) The cytotoxicity of 2-NapGFFT peptides to $\mathrm{L}^{-\mathrm{O}_{2}}$ cells. (B) The cytotoxicity of 2-NapGFFTT peptides to $\mathrm{L}-\mathrm{O}_{2}$ cells. (C) The cytotoxicity of 2-NapGFFD peptides to $\mathrm{L}_{-} \mathrm{O}_{2}$ cells. 

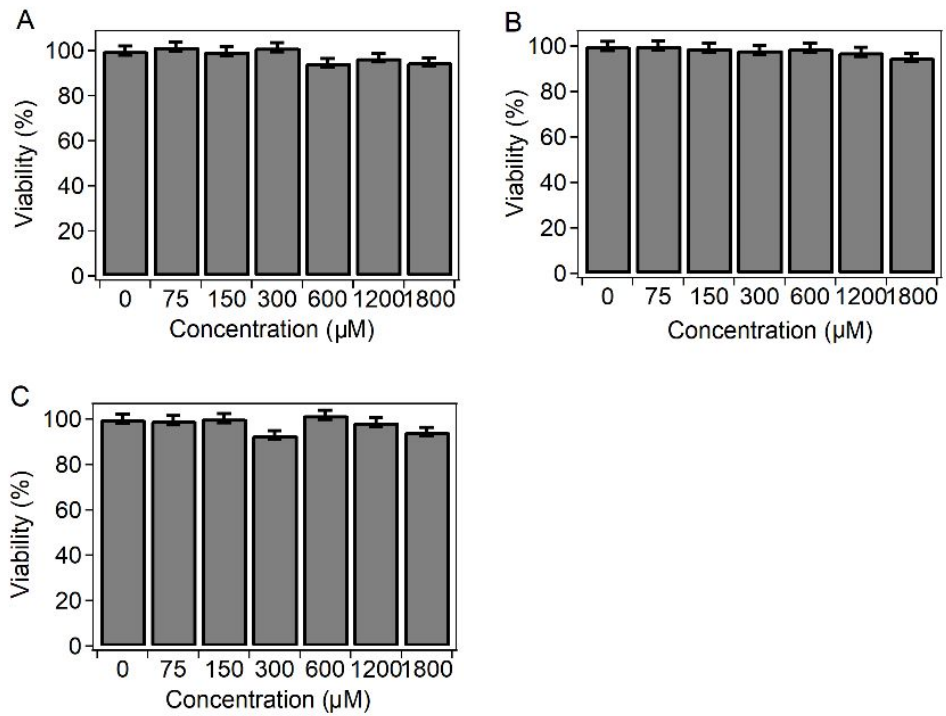

Figure S8 (A) The cytotoxicity of 2-NapGFFT peptides to SMMC-7721 cells. (B) The cytotoxicity of 2NapGFFTT peptides to SMMC-7721 cells. (C) The cytotoxicity of 2-NapGFFD peptides to SMMC-7721 cells.
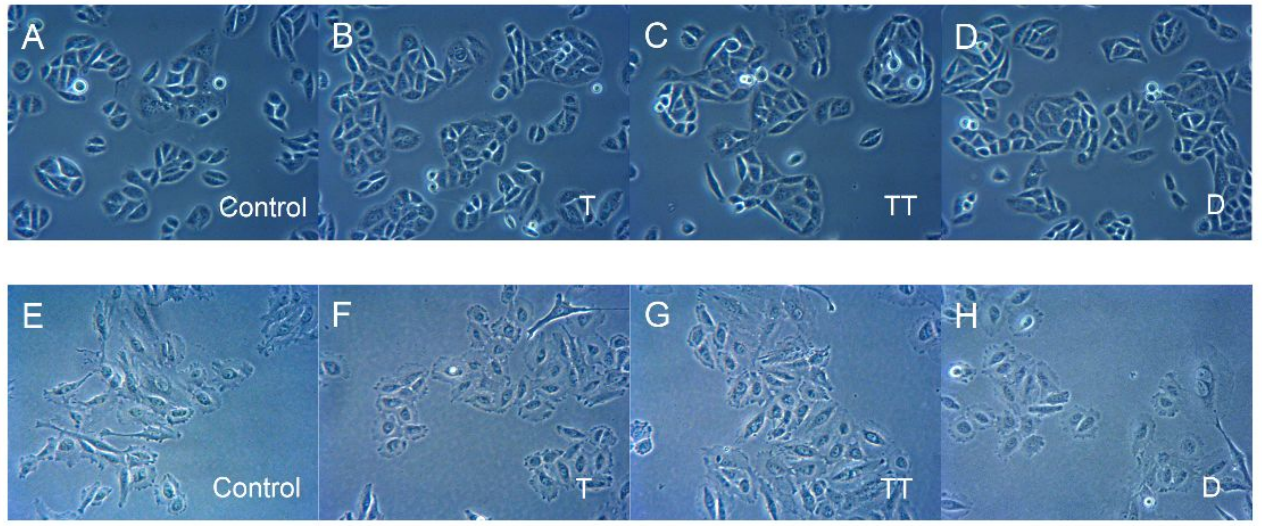

Figure S9 The cytotoxicity of the peptides to LO-2 and SMCC-7721 cells. (A) Optical image of LO-2 cells cultured without peptides. (B) Optical image of $L O-2$ cells cultured with 2-NapGFFT peptides (30o $\mu \mathrm{M})$. (C) Optical image of $L O-2$ cells cultured with 2-NapGFFTT peptides (300 $\mu \mathrm{M})$. (D) Optical image of $L O-2$ cells cultured with 2-NapGFFD peptides $(300 \mu \mathrm{M})$. (E) Optical image of SMMC-7721 cells cultured without peptides. (F) Optical image of SMMC-7721 cells cultured with 2-NapGFFT peptides (300 $\mu \mathrm{M})$. (G) Optical image of SMMC-7721 cells cultured with 2-NapGFFTT peptides (300 $\mu \mathrm{M})$. (H) Optical image of SMMC-7721 cells cultured with 2-NapGFFD peptides (300 $\mu \mathrm{M})$. T, TT and D correspond to 2-NapGFFT, 2-NapGFFTT and 2-NapGFFD peptides, respectively. 

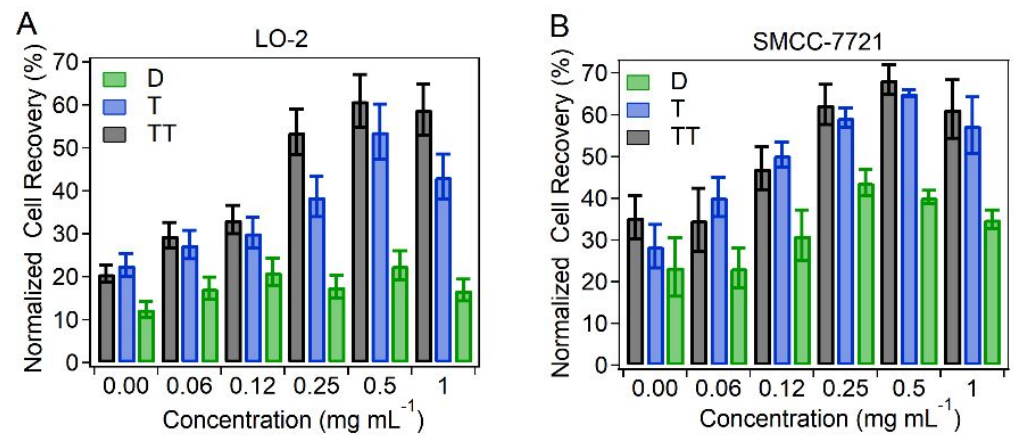

Figure S1o The normalized cell recovery of L-O2 (A) and SMMC-7721 (B) cells protected with different concentrations of peptides after 24 hours post-thaw growth. Cells protected with peptides after the freezethaw cycle and fresh cells without frozen were allowed to growth for 24 hours. The $\%$ of living cells protected with peptides relative to the fresh cells were reported. Error bars are S.D. from 3 repeats.
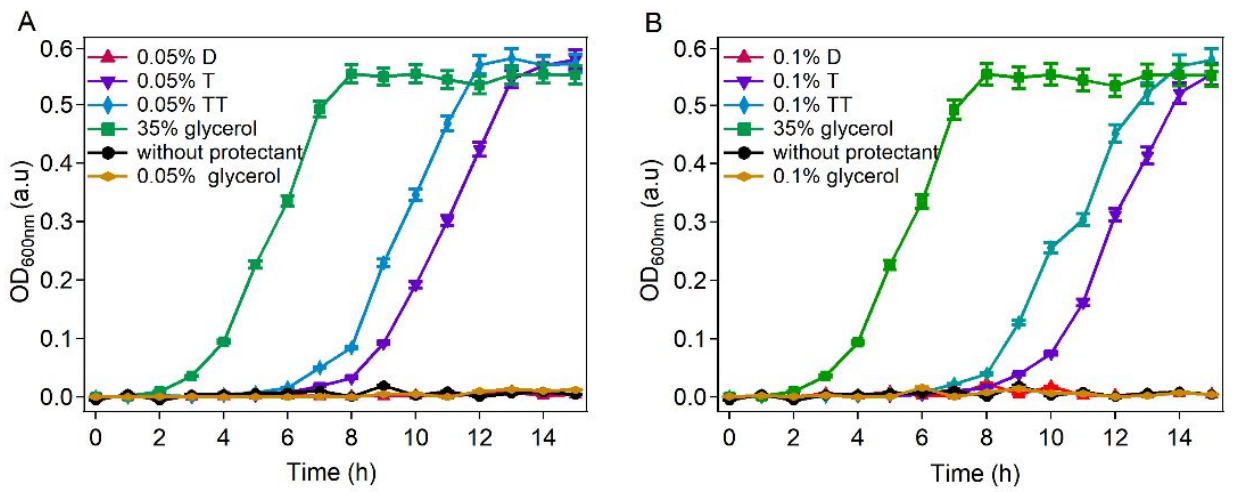

Figure S11 (A) The growth curves of E.coli in PBS (10 mM, pH = 7.4) containing different peptides at the concentration of $0.05 \% \mathrm{w} / \mathrm{v}$. (B) The growth curves of E.coli in PBS (10 mM, pH=7.4) containing different peptides at the concentration of $0.1 \% \mathrm{w} / \mathrm{v}$. T, TT and D correspond to 2-NapGFFT, 2-NapGFFTT and 2NapGFFD peptides, respectively.

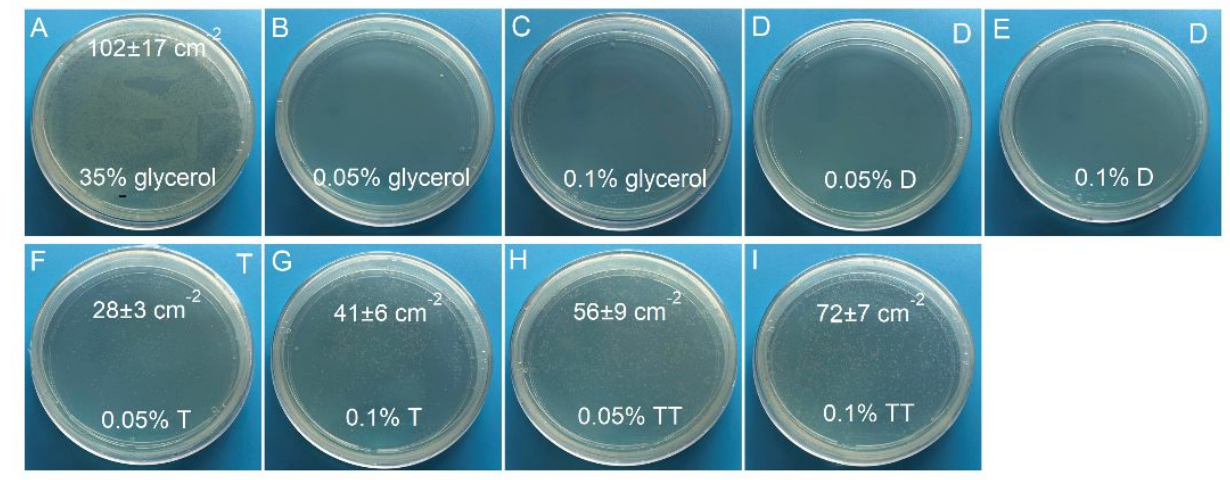

Figure S12 Cryoprotection of bacteria using self-assembling peptides. T, TT and D correspond to 2-NapGFFT, 2-NapGFFTT and 2-NapGFFD peptides, respectively. The mixtures of $E$. coli with different cryoprotectants in PBS (10 mM, pH = 7.4) were treated by three freeze-thaw cycles before being inoculated to each agar plate. (A-C) Glycerol at mass concentrations of 35\%, 0.05\% and 0.1\%, respectively. (D-E) 2-NapGFFD at mass concentrations of $0.05 \%$ and $0.1 \%$, respectively. (F-G) 2-NapGFFT at mass concentrations of $0.05 \%$ and $0.1 \%$, 
respectively. (H-I) 2-NapGFFTT at mass concentrations of $0.05 \%$ and $0.1 \%$, respectively. The average density of the colonies on three plates were indicated on the figures.

\section{Reference}

(1) Murray, B.; O'sullivan, D.; Atkinson, J.; Webb, M. Ice nucleation by particles immersed in supercooled cloud droplets. Chem. Soc. Rev. 2012, 41, 6519. 